\title{
Lifestyle and physical health in schizophrenia
}

\author{
Moira Connolly \& Ciara Kelly
}

\begin{abstract}
People with schizophrenia die prematurely. Their illness, its treatment and their lifestyle all contribute to the excess morbidity and mortality. Lifestyle 'choices' (e.g. poor diet, low rates of physical activity and increased likelihood to smoke cigarettes) predispose them to poor physical health and comorbid medical diseases. In addition, weight gain and obesity are a consequence of most antipsychotics, particularly the atypicals. Excessive body weight increases the risk of morbidity and mortality, and is the biggest risk factor for type II diabetes in schizophrenia. Much of the excess mortality of schizophrenia is preventable through lifestyle and risk factor modification and the treatment of common diseases, but the potential for improving outcomes in this area is only starting to be addressed.
\end{abstract}

This article is the second of two in this issue in a series introduced by Robin McCreadie's editorial 'Schizophrenia revisited' (McCreadie, 2004). In the first, Kerwin E Bolonna (2005) discuss treatment-resistant schizophrenia. Previous contributions have considered environmental influences (Leask, 2004), implementation of the NICE schizophrenia guidelines (Rowlands, 2004), cognitive deficits in first-episode schizophrenia (Gopal E Variend, 2005) and early intervention in psychosis (Singh E Fisher, 2005).

People with schizophrenia have a higher risk of medical illness than does the general population. They also have an increased (by two- to fourfold) relative risk of premature death, dying at least 10 years earlier than age-matched contemporaries (Brown, 1997; Joukamaa et al, 2001). Brown's study of mortality in schizophrenia found that $12 \%$ of reported deaths were by suicide, which accounted for $28 \%$ of the excess. A further two-thirds of this excess was attributed to 'natural causes' such as cardiovascular disease, respiratory disease and diabetes.

Undoubtedly, much of the excess morbidity and mortality of schizophrenia is preventable through lifestyle modification and the recognition and treatment of common diseases. Although there is a clear evidence base acknowledging the poor physical health of people with mental illness, the potential for improving outcomes in this area has been largely neglected in both research and clinical practice.

With changing clinical practice, outcome studies have tended to concentrate on psychiatric outcomes - symptomatology, service use, level of function and quality of life. The general lifestyle and physical health of patients with schizophrenia are relatively rarely studied.

In this article, we bring together these areas of research and practice to inform the debate on how to improve the physical health of patients with schizophrenia and, ultimately, overall outcomes for the illness.

\section{Risk factors}

Morbidity can be estimated individually using predictive risk factors and markers, some clearly modifiable and others not (Box 1). Modifiable risk factors are prevalent in people with schizophrenia. They are a consequence of the illness itself, and of associated behaviours, lifestyle and treatment. It is clearly important that services available to people with schizophrenia appreciate the inflated risk and how it contributes to morbidity and premature mortality in these individuals.

\section{Lifestyle}

Our lifestyle choices are influenced by a variety of factors such as genes, environment and sociodemographic status. In schizophrenia, the illness itself also plays a part. A drift down the social scale associated with unemployment and poorer financial

Moira Connolly is a consultant general psychiatrist at Gartnavel Royal Hospital (Great Western Road, Glasgow G12 0XH, UK. E-mail: Moira.Connolly@glacomen.scot.nhs.uk) with interests in psychiatric rehabilitation and cognitive-behavioural therapy. She is an honorary senior lecturer at the Department of Psychological Medicine, University of Glasgow, where her research interests include the physical health of people with schizophrenia. Ciara Kelly is a consultant psychiatrist working in rehabilitation at Leverndale Hospital, Glasgow, with academic time spent in the Department of Psychological Medicine, Glasgow University. Her current research interests are the lifestyle, physical health and outcome of people with schizophrenia. 


\section{Box 1 A brief explanation of risk}

- A risk factor is a manifestation or a laboratory measurement that expresses the likelihood that an individual or a group will develop a disease over a defined period of time

- A risk factor may play a causal role in the pathogenesis of a disease or it may be an associated marker. For example,low-density lipid (LDL) cholesterol is a risk factor in coronary heart disease proven to be modifiable in intervention trials, whereas C-reactive protein is a risk marker - it is associated with coronary heart disease but is not necessarily causal

- Specific diseases can have both modifiable and non-modifiable risk factors. For example, modifiable risk factors for coronary heart disease include obesity, dyslipidaemia, diabetes, hypertension, smoking and psychological state

standing once the illness is established is not unusual. It is also hypothesised that an 'urban risk factor' operates in the aetiology of schizophrenia, increasing its incidence (Jablensky, 1999).

Two fairly recent studies (Brown et al, 1999; McCreadie, 2003) have compared the lifestyle of people with schizophrenia living in the community with that of low social class cohorts from existing general population studies of lifestyle habits. In both studies, people with schizophrenia made significantly poorer dietary choices, took less exercise and smoked more heavily than the comparator groups in the general population. McCreadie also found that women with schizophrenia were significantly more likely to be overweight or obese than women in the general population (a result not found by Brownet al).

Given that poor diet, smoking and excess weight are potentially modifiable factors associated with increased physical morbidity and mortality, we will consider further the extent of these problems in patients with schizophrenia.

\section{Diet}

A small case-control study in a Scottish population demonstrated that people with schizophrenia made poor dietary choices, characterised by high fat and low fibre intake (McCreadie et al, 1998). The subsequent larger study (McCreadie, 2003) examined in detail the dietary intake of 102 people with schizophrenia in Dumfries and Glasgow. Their fruit and vegetable consumption averaged 16 portions per week (less than half the recommended intake), and very few patients made acceptable dietary choices across a range of foodstuffs. Vitamin C levels correlated with fruit and vegetable intake, carotenoid levels were indicative of a diet containing little in the way of fruit and vegetables, and folate deficiency was detected in $2 \%$ of patients.

It is clear that the diet of people with schizophrenia in Scotland is at least as poor as that of the general population in social class $\mathrm{V}$ (the lowest social class). In a nation whose dietary habits are among the poorest in Europe, this is concerning.

Minimal consideration has been given to explanatory factors for the poor diet of this patient group, and dietary interventions have tended to centre on the impact of vitamins or omega- 3 fatty acid supplementation on mental health, with varying outcomes (Joy et al, 2003).

\section{Physical activity}

Brown et al (1999) and McCreadie (2003) found that people with schizophrenia tended to take only small amounts of exercise. The reason for this has not been demonstrated, but factors such as features of the illness, sedative medication and lack of opportunity and general motivation may be relevant.

The relative risk of atherosclerosis in physically inactive individuals is higher than in those who are more active. The specific mechanism by which physical activity reduces mortality from cardiovascular disease is unknown, but exercise has been shown to improve lipid profiles, glucose tolerance, obesity and hypertension.

\section{Smoking}

It has been suggested that smoking represents the most extensively documented cause of disease ever investigated in the history of biochemical research. Smoking remains the single greatest preventable cause of death in our society.

A review of smoking and schizophrenia published in a previous issue of APT (Kelly \& McCreadie, 2000) noted the following findings.

The prevalence of smoking in schizophrenia greatly exceeds that in the general population (75$92 \%$ v. 30-40\%). Furthermore, heavy cigarette smoking is intimately associated with schizophrenia and it may have implications for the underlying neurobiology of the disease. Patients who smoke report increased cigarette consumption, are more addicted to nicotine and have higher nicotine levels in the bloodstream. Smoking may be a marker for a more severe illness. Cigarette smoking induces 
hepatic microsomal enzymes, which increase the metabolism of psychotropic medication; therefore smokers usually require greater levels of antipsychotic medication than non-smokers to achieve similar blood levels.

Most heavy smokers find it very difficult to give up, and success rates are even lower in people with psychiatric illness. Attempts to get patients with schizophrenia to stop smoking have met with variable success. It is widely believed (by mental health professionals, families, carers and patients themselves) that it is one of the patients' few pleasures, that it is 'hopeless' to try to quit and that to do so will aggravate their mental state. This view is discriminatory. In a survey of cigarette-smoking patients, one-third reported that they wanted to quit for health reasons. Clearly, smokers with schizophrenia have a severe nicotine addiction, and pharmacological and psychological support with cessation needs to address their particular needs. There is some evidence that clozapine, and possibly other atypicals, reduce cigarette consumption.

\section{Obesity}

Although often not viewed as a health problem, being overweight or obese (Box 2) has reached epidemic proportions worldwide. Excessive body weight substantially increases the risk of morbidity from a number of conditions, including hypertension, dyslipidaemia, type II diabetes mellitus, coronary heart disease, stroke, gallbladder disease, osteoarthritis, sleep apnoea, respiratory problems and endometrial, breast, prostate and colon cancers. Higher body weight is associated with increased mortality as well as with social stigmatisation (Meltzer \& Fleischhacker, 2001). Excess abdominal fat is

\section{Box 2 Body weight and obesity}

The body mass index (BMI)

Determined as weight divided by the square of height $\left(\mathrm{kg} / \mathrm{m}^{2}\right)$, BMI is one measure of body weight. 'Overweight' is defined as BMI $\geq 25 \mathrm{~kg}$ / $\mathrm{m}^{2}$ and 'obese' as BMI $\geq 30 \mathrm{~kg} / \mathrm{m}^{2}$

\section{Waist circumference}

This is another useful measure because it is an indicator of the amount of abdominal fat. It is easy and cheap and is highly correlated with specific measures of abdominal fat including more complex imaging

Waist-to-hip ratio

Expression of abdominal fat in this form is no longer considered necessary (Ryan et al, 2003)
Box 3 Effects of weight gain in people with schizophrenia (Tardieu et al, 2003)

- Health risks

- hypertension

- atherosclerosis

- type II diabetes mellitus

- cardiovascular disease and stroke

- Stigmatisation

- Non-adherence to treatment

- Further impairment of quality of life

- Social withdrawal

associated with dyslipidaemia, hypertension and glucose intolerance. Risk of comorbid diseases has been shown to rise as BMI increases above $25 \mathrm{~kg} / \mathrm{m}^{2}$ and waist circumference increases above $102 \mathrm{~cm}$ for men or $89 \mathrm{~cm}$ for women (Aronne, 2001). Increased rates of diabetes have also been shown to be related to body fat distribution (Ryan \& Thakore, 2002).

In psychiatric practice, weight gain is a longrecognised and commonly encountered problem. In a review of the literature, Allison \& Casey (2001) report on a study that used the 1989 US National Health Interview Survey data comparing weights and heights of people with and without schizophrenia. In general, the results showed that those with schizophrenia were more obese than those without, and that the difference was significant for women. Another study reviewed by Allison \& Casey, of 151 people with schizophrenia, reported $51 \%$ of males and $59 \%$ of females to be clinically obese, compared with $33 \%$ of people with other psychiatric disorders.

\section{The role of antipsychotics}

Antipsychotic-related weight gain was first reported in association with chlorpromazine in the late 1950s, but it has remained overshadowed by other sideeffects such as extrapyramidal symptoms and tardive dyskinesia. Among the conventional antipsychotics, weight-gain liability appears to be greatest with low-potency drugs. Meta-analyses, clinical trials and clinical experience suggest that the atypical antipsychotics can also cause marked weight gain during treatment. Box 3 outlines some of the consequences of this problem.

Not all antipsychotics have the same propensity for causing weight gain (Box 4), but those associated with the greatest gain, clozapine and olanzapine, can add up to $4.5 \mathrm{~kg}$ after 10 weeks of treatment at standard dose. Also, patients appear to differ in their weight-gain response to specific drugs. Little is known about individual predictors, but most weight gain appears to occur during the first 2 years of treatment, and it accompanies an increase in 
Box 4 Effect on weight of different antipsychotics (after Zimmerman et al, 2003)

Marked weight gain

- Clozapine

- Olanzapine

- Zotepine

- Quetiapine

- Chlorpromazine

- Thioridazine

- Perphenazine

- Trifluoperazine

Moderate weight gain

- Risperidone

- Clopenthixol

- Sulpiride

Slight weight gain

- Amisulpride

- Haloperidol

- Fluphenazine

- Flupentixol

No weight change

- Ziprasidone

Weight loss

- Molindone

- Pimozide

No data available

- Aripiprazole

appetite, especially for sweet and fatty foods, with no clear impact on basal metabolic rate.

Fontaine et al (2001) estimated the consequences of antipsychotic-induced weight gain ( 0 to $12.75 \mathrm{~kg}$ ) on selected mortality rates and the incidence of diabetes and hypertension in US adults. For people who moved into the overweight and obese BMI categories, the gain was expected to result in 258 deaths per 100000 people over 10 years. A gain of $2.5 \mathrm{~kg}$ was expected to result in 350 cases of impaired glucose tolerance and 1850 cases of hypertension per 100000 people.

These figures are of profound clinical and public health significance. Of particular interest in relation to current health policy are Fontaine et al's conclusions regarding suicide rates. The government of England and Wales has set mental health services the target of reducing suicides by at least one-fifth by 2010 (Department of Health, 1999). At present, 10-13\% of people with schizophrenia die by suicide. Fontaine et al estimated that the use of clozapine for schizophrenia over a 10-year period would prevent 492 deaths from suicide per 100000 patients; unfortunately, these would be offset by 416 deaths due to a presumed clozapine-induced weight gain of $10 \mathrm{~kg}$.

\section{Management}

Managing obesity in people with schizophrenia can be daunting. Compared with non-obese patients, obese patients are 13 times more likely to request discontinuation of their current antipsychotic medication because of concerns about weight gain and 3 times more likely to fail to adhere to treatment regimens (Kurzthaler \& Fleischhacker, 2001).

Weight-loss interventions for people with schizophrenia should start with regular and frequent weight monitoring and should advise on exercise and lifestyle. Switching of antipsychotic medication to one with less propensity for weight gain should also be considered.

In general, pharmacotherapy should be reserved for obese patients who fail to lose weight after several months of behavioural interventions, and particularly for those with comorbid disorders such as diabetes. One of the new anti-obesity drugs orlistat, a lipase inhibitor, may be the best choice for the treatment of obesity in people with chronic mental illness. Long-term general population studies show sustained weight loss of $9.7 \%$ at 2 years with a dose of $120 \mathrm{mg}$ thrice daily with meals. Improvements in fasting lipid profiles and glucose tolerance were noted. There have been case reports of its safe and effective use in psychiatric patients, with no effects on the bioavailablity of psychotropic medication. However, to date there have been no randomised controlled trials of its use in such populations (Hilger et al, 2002).

\section{Lipids}

Elevated blood lipids, particularly triglycerides, are associated with some typical antipsychotic agents. Shortly after their introduction, phenothiazines were found to elevate serum triglyceride and total cholesterol levels. This compared with a minimal or slightly favourable effect seen with butyrophenones.

Much has been written on the effects of specific atypical drugs on lipid profiles. Both clozapine and olanzapine have been shown to cause significant hypertriglyceridemia compared with typicals. Some studies have reported a significant association between weight gain and triglyceride change for patients receiving atypical antipsychotic therapy, but other studies suggest a direct effect of clozapine and olanzapine on lipid levels not associated with weight gain (Meyer, 2001).

The exact biochemical locus at which atypicals exert their influence on triglyceride metabolism remains a source of speculation. However, it has been noted that the atypicals that exert significant effects on fasting triglyceride levels are dibenzodiazepinederived compounds - clozapine, olanzapine and 
quetiapine. They have the propensity to increase serum triglyceride levels with lesser effects on cholesterol (Meyer, 2001).

\section{Diabetes}

Diabetes is a growing problem in the general population: type II diabetes mellitus currently affects about $2 \%$ of people in the UK. Risk factors for the disorder are shown in Box 5. Type II diabetes (non-insulin dependent) is associated with decreased sensitivity to the actions of insulin (insulin resistance), together with a variable and usually progressive defect in $\beta$ cell function leading to a relative insulin deficiency. Type I diabetes mellitus is a primary $\beta$-cell defect causing failure to secrete insulin - a condition not generally associated with schizophrenia.

Diabetic ketoacidosis is an uncommon complication of diabetes mellitus; more common complications include microvascular disease (e.g. retinopathy, nephropathy, neuropathies) and macrovascular disease (e.g. cardiovascular and cerebrovascular disease). Hyperglycaemia involving glucose levels low enough to fall below the diagnostic threshold for diabetes has been associated with increased vascular morbidity and mortality in patients treated with antipsychotics (Newcomer et al, 2001).

\section{Diabetes in schizophrenia}

Hyperglycaemia is an underrecognised comorbid complication of treatment with antipsychotic medication and it may contribute to the increased morbidity and mortality seen in schizophrenia (Tardieu et al, 2003). Dixon et al (2000), reporting

\section{Box 5 Risk factors for type II diabetes}

Intrinsic risk factors

- Family history

- Age greater than 45 years

- Ethnicity (increased risk for Black and minority ethnic groups)

- Previous history of glucose intolerance

Modifiable risk factors

- Dyslipidaemia

- Lack of exercise

- Hypertension

- Obesity

Less-well established risk factors

- Serious psychiatric illness

- Alcohol misuse

- Diet

- Hyperprolactinaemia on the USA's Schizophrenia Patient Outcomes Research Team (PORT) and National Health Interview Survey, studied the prevalence and correlates of diabetes in people with schizophrenia. The PORT survey interviewed 719 people with schizophrenia (mean age 43 years) about their physical health between 1991 and 1996. It found 14.9\% life-time diabetes and $10.8 \%$ self-reported current diabetes. This compared poorly with general population data from the National Health Interview Survey, in which diabetes rates in the general population were $1.2 \%$ (18- to 44-year-olds) and 6.3\% (45- to 64-year-olds). Thus, in the USA in the mid-1990s (probably before the widespread use of atypical antipsychotics) the rate of diabetes in people with schizophrenia already exceeded that in the general population.

Two more studies echo these results. Ryan et al (2003) reported that more than $15 \%$ of their sample of drug-naïve patients with first-episode schizophrenia had impaired fasting glucose compared with none of the matched healthy volunteers. In addition, a small study by Mukherjee et al (1996) reported a family history of type II diabetes in 18$19 \%$ of their sample of people with schizophrenia.

\section{Risk factors}

The biggest risk factor for emergent diabetes in schizophrenia is weight gain caused by antipsychotics. This has been clearly described for conventional antipsychotics and has become a focus of much investigation for the atypical agents (Sussman, 2001).

It is possible that other factors are associated with an increased prevalence of type II diabetes in people with schizophrenia; these might include diet, lifestyle and behaviour, as well as a genetic predisposition that may be shared with the disorder itself.

Since 1994, several case reports and uncontrolled studies have linked the atypical antipsychotic clozapine with the development of diabetes mellitus. A recent naturalistic uncontrolled follow-up study of 82 people (mean age 36.4 years; mean BMI $=26.9$ ) starting clozapine reported that $36.6 \%(30 / 82)$ developed diabetes during the first 5 years of treatment (Henderson et al, 2000).

It has been hypothesised that diabetes occurs secondary to the often substantial weight gain with antipsychotics. However, it seems that the atypical antipsychotics may also contribute more directly to the pathogenesis of hyperglycaemia. There have been several case reports and warnings by the UK's Committee on Safety of Medicines associating olanzapine with acute-onset diabetes. Most, but interestingly not all of these patients experienced significant weight gain shortly after commencing treatment. Of note, a case series of four patients reported reduced glycaemic control with clozapine 


\section{Box 6 Core features of metabolic syndrome}

- Obesity - central or upper body

- Insulin resistance or hyperinsulinaemia

- Dyslipidaemia

- Impaired glucose tolerance or type II diabetes mellitus

- Hypertension

not related to weight gain. One study of patients on antipsychotics showed that 29 out of the 30 cases of diabetes diagnosed were identified at a routine annual screening of fasting plasma glucose, illustrating the need for regular biochemical monitoring (Meyer, 2001).

Diabetes is a chronic disease that requires active self-care for optimal management; in view of the cognitive, social and psychological disabilities of people with schizophrenia, optimal care is often unlikely.

\section{Metabolic syndrome}

In a detailed review by Ryan \& Thakore (2002), evidence is presented suggesting that aspects of metabolic syndrome (Box 6) exist in patients with schizophrenia. They highlight the potential role of visceral fat as a common pathological factor in explaining some types of cancer, cardiovascular illness, type II diabetes mellitus and dyslipidaemia in patients with schizophrenia. In a subsequent study, Thakore and colleagues report findings that drug-naïve and drug-free patients had more than three times the visceral fat found in matched controls using computed tomography (Thakore et al, 2002). By comparison, in a controlled study of drug-naïve Chinese patients that used magnetic resonance imaging, Zhang et al (2004) found only slight elevations in fat indicators at baseline, but significantly increased subcutaneous and intraabdominal fat following 10 weeks of treatment mostly with chlorpromazine and risperidone

Lifestyle factors associated with metabolic syndrome (such as increased dietary fat content, reduced physical activity, smoking, excess alcohol consumption and other endocrine and cardiovascular factors) are clearly in evidence in some patients with schizophrenia.

\section{Prolactin elevation}

Conventional antipsychotics and, of the atypicals, risperidone in particular, can have deleterious effects on sexual and endocrine function (Meaney \& O'Keane, 2002). Raised prolactin levels reduce pituitary sensitivity to gonadotropin-releasing hormone in women, affecting reproductive function and causing oligo- and amenorrhoea. Hypooestrogenism comparable to postmenopausal status can occur and may predispose women to cardiovascular disease. In men there is reduced steroidogenesis and spermatogenesis.

Reduced bone mineral density in patients with hyperprolactinaemia may be caused directly or (due to hypogonadism) indirectly. Risk factors for the development of osteoporosis and the potential impact of antipsychotics have been reviewed by Naidoo et al (2003) and are outlined in Box 7. Peak bone mass is established in the first two decades of life, and antipsychotic medication at this stage could therefore have long-lasting effects on bone mineral density.

\section{Morbidity and mortality in schizophrenia}

The impact of deinstitutionalisation on mortality rates has been studied in Norway (Hansen et al, 2001) and Finland (Salokangas et al, 2002). In Norway, allcause mortality rates for people with functional psychosis increased significantly between 1980 and 1992 (compared with the 1950s and 1960s), the period during which deinstitutionalisation took place. In Finland, however, although mortality rates were higher than population norms for the study period of 1982 to 1994 (during which time psychiatric beds were more than halved), there was no increased mortality for patients with schizophrenia. No comparison was made with earlier time periods.

Box 7 Potential risk factors for osteoporosis in schizophrenia (after Naidoo et al, 2003)

Factors due to schizophrenia itself

- Poor diet

- Limited weight-bearing exercise

- Smoking (has a direct toxic effect on osteoblasts)

- Polydipsia

Factors due to antipsychotic treatment

- Hyperprolactinaemia

- Secondary lowered oestrogen

- Secondary lowered testosterone

Other factors influencing risk of fracture

- Sedation

- Orthostatic hypotension

- Dizziness and falls

- Postmenopausal status

- Anticonvulsants

- Corticosteroids 
The relationship between quality of care and mortality rates for cardiovascular disease in older patients with schizophrenia has been studied by Druss et al (2001). They have shown that the excess mortality rates for these patients after myocardial infarction were reduced in magnitude and became non-significant when adjustment was made for the presence or absence of quality measures such as reperfusion therapy, use of aspirin, beta-blockers or angiotensin-converting enzyme (ace) inhibitors, and smoking cessation therapy. The study does not clarify whether differences in the provision of quality of care to these psychiatric patients are due to patient or provider factors.

Psychotropic medication in the treatment of schizophrenia can exert a number of cardiovascular effects. The safer selection of these drugs for vulnerable patients has been the subject of a previous APT review (O’Brien \& Oyebode, 2003).

\section{The future}

In considering what to do about the poor physical health of people with schizophrenia, we must first take account of their own opinions. Many rate physical health as a high priority and the more physical conditions they have, the poorer their satisfaction with their physical and mental health (Dixon et al, 1997).

That the excess mortality from natural causes in schizophrenia may be contributed to by an unhealthy lifestyle and might therefore be amenable to health promotional interventions is not in dispute. But inducing change may not be easy. The extent of the contribution of the illness itself (either through positive, negative or cognitive symptoms) and of any biological predisposition is not clear, but is not so small as to be rendered insignificant. Furthermore, the additional risks to physical health potentially induced by the complex interplay of medication and specific predispositions to metabolic disorders cannot be ignored. Psychiatric training may not equip psychiatrists to adequately examine and investigate the physical health of their patients. ${ }^{\dagger}$ However, it remains the psychiatrists' responsibility to be aware of the health consequences of psychiatric disorder and the treatments they initiate.

People with schizophrenia may fail to recognise the early signs of physical ill health or avoid contact with health services. The traditional routes to obtaining healthcare may prove too complex for someone who is chronically distressed by positive symptoms, cognitively impaired or lacking the motivation required to make and attend an appointment.

${ }^{\dagger}$ For a discussion of shared care for people with mental illness see pp. 133-141, this issue. Ed.
So what of the future? Our view is that the evaluation of new therapies should include detailed assessments of physical health and future risk estimates in addition to standard psychiatric outcomes. Psychiatrists must ensure that they arrange the appropriate examination and investigation of patients at risk of developing significant physical morbidity, working closely with general practitioners and with other specialists when appropriate. The advantages of atypical drugs are clear, but psychiatrists should weigh up the risks of metabolic disturbance and its potential impact on future cardiovascular risk when selecting an antipsychotic for a specific patient. They should take a careful medical history and be prepared to monitor weight and other metabolic markers (such as glucose and lipid profile) over time. This information will help clinicians analyse the risks and benefits of a specific treatment for an individual patient.

The challenge for all is to ensure that the physical health of patients with schizophrenia is given the priority it deserves, helping them to face their future with the lowest possible morbidity and mortality odds stacked against them.

\section{References and further articles}

Allison, D. B. \& Casey, D. E. (2001) Antipsychotic-induced weight gain. A review of the literature. Journal of Clinical Psychiatry, 62 (suppl. 7), 22-31.

Aronne, L. J. (2001) Epidemiology, morbidity, and treatment of overweight and obesity. Journal of Clinical Psychiatry, 62 (suppl. 23), 13-22.

Brown, S. (1997) Excess mortality of schizophrenia. A metaanalysis. British Journal of Psychiatry, 171, 502-508.

Brown, S., Birtwistle, J., Roe, L. et al (1999) The unhealthy lifestyle of people with schizophrenia. Psychological Medicine, 29, 697-701.

Department of Health (1999) Saving Lives: Our Healthier Nation. London: Stationery Office.

Dixon, L., Postrado, L., Delahauty, J., et al (1997) The association of medical co-morbidity in schizophrenia with poor physical and mental health. Journal of Nervous and Mental Disease, 187, 496-502.

Dixon, L., Weiden, P., Delahanty, J., et al (2000) Prevalence and correlates of diabetes in national schizophrenia samples. Schizophrenia Bulletin, 26, 903-912.

Druss, B. G., Bradford, W. D., Rosenheck, R. A., et al (2001) Quality of medical care and excess mortality in older patients with mental disorders. Archives of General Psychiatry, 58, 565-572.

Fontaine, K. R., Moonseong, H., Harrigan, E. P., et al (2001) Estimating the consequences of antipsychotic induced weight gain on health and mortality rate. Psychiatric Research, 101, 277-288.

Gopal, Y. V. \& Variend, H. (2005) First-episode schizophrenia: review of cognitive deficits and cognitive remediation. Advances in Psychiatric Treatment, 11, 38-44.

Hansen, V., Jacobsen, B. K. \& Arnesen, E. (2001) Cause-specific mortality in psychiatric patients after deinstitutionalisation. British Journal of Psychiatry, 179, 438-443.

Henderson, D. C., Cagliero, E., Gray, C., et al (2000) Clozapine, diabetes mellitus, weight gain, and lipid abnormalities.A five-year naturalistic study. American Journal of Psychiatry, 157, 975-981.

Hilger, E., Quiner, S., Ginzel, I., et al (2002) The effects of orlistat on plasma levels of psychotropic drugs in patients 
with long-term psychopharmacotherapy. Journal of Clinical Psychopharmacology, 22, 68-70.

Jablensky, A. (1999) Schizophrenia: epidemiology. Current Opinion in Psychiatry, 12, 19-28.

Joukamaa, M., Heliovaara, M., Knekt, P., et al (2001) Mental disorders and cause specific mortality. British Journal of Psychiatry, 179, 498-502.

Joy, C. B., Mumby-Croft, R. \& Joy, L. A. (2003) Polyunsaturated fatty acid supplementation for schizophrenia. Cochrance Library, issue 2. Oxford: Update Software.

Kelly, C. \& McCreadie, R. (2000) Cigarette smoking and schizophrenia. Advances in Psychiatric Treatment, 6, 327-332.

Kerwin, R. W. \& Bolonna, A. (2005) Management of clozapineresistant schizophrenia. Advances in Psychiatric Treatment, 11, 101-106.

Kurzthaler, M. D. \& Fleischhacker, W. W. (2001) The clinical implications of weight gain in schizophrenia. Journal of Clinical Psychiatry, 62 (suppl. 7), 32-37.

Leask, S. J. (2004) Environmental influences in schizophrenia: the known and the unknown. Advances in Psychiatric Treatment, 10, 323-330.

McCreadie, R. G. (2003) Diet, smoking and cardiovascular risk in people with schizophrenia. Descriptive study. British Journal of Psychiatry, 183, 534-539.

McCreadie, R. G. (2004) Editorial: Schizophrenia revisited. Advances in Psychiatric Treatment, 10, 321-322.

McCreadie, R. G., MacDonald, E., Blacklock, C., et al (1998) Dietary intake of schizophrenia patients in Nithsdale, Scotland: Case Control Study. BMJ; 317, 784-785.

Meaney, A. M. \& O'Keane, V. (2002) Prolactin and schizophrenia. Clinical consequences of hyperprolactinaemia. Life Sciences, 71, 979-992.

Meltzer, H. Y. \& Fleischhacker, W. W. (2001) Weight gain: a growing problem in schizophrenia management. Journal of Clinical Psychiatry, 62 (suppl. 7), 1-43.

Meyer, J. M. (2001) Effects of atypical antipsychotics on weight and serum lipid levels. Journal of Clinical Psychiatry, 62 (suppl. 27), 27-34.

Mukherjee, S., Decins, P., Bocola, V., et al (1996) Diabetes mellitus in schizophrenic patients. Comprehensive Psychiatry, $37,68-73$.

Naidoo, U., Goff, D. C. \& Klibanski, A. (2003) Hyperprolactinaemia and bone mineral density: the potential impact of antipsychotic agents. Psychoneuroendocrinology, 28 (suppl. 2), 97-108

Newcomer, J. W., Haupt, D. W., Fucetola, R., et al (2001) Metabolic disturbances associated with antipsychotic use. Journal of Clinical Psychiatry, 61 (suppl. 27), 1-43.

O'Brien, P. \& Oyebode, F. (2003) Psychotropic medication and the heart. Advances in Psychiatric Treatment, 9, 414-423.

Rowlands, P. (2004) The NICE schizophrenia guidelines: the challenge of implementation. Advances in Psychiatric Treatment, 10, 403-412.

Ryan, M. C. M. \& Thakore, J. H. (2002) Physical consequences of schizophrenia and its treatment. The metabolic syndrome. Life Sciences, 71, 239-257.

Ryan, M. C. M., Collins, P. \& Thakore, J. H. (2003) Impaired fasting glucose tolerance in first episode, drug naïve patients with schizophrenia. American Journal of Psychiatry, 160, 284289.

Salokangas, R. K., Honkonsen, T., Stengard, E., et al (2002) Mortality in chronic schizophrenia during decreasing number of psychiatric beds in Finland. Schizophrenia Research, 54, 265-275.

Singh, S. P. \& Fisher, H. L. (2005) Early intervention in psychosis: obstacles and opportunities. Advances in Psychiatric Treatment, 11, 71-78.

Sussman, N. (2001) Review of atypical antipsychotics and weight gain. Journal of Clinical Psychiatry, 62 (suppl. 23), 512 .

Tardieu, S., Micallef, J., Gentile, S., et al (2003) Weight gain profiles of new anti-psychotics: public health consequences. Obesity Reviews, 4, 129-138.

Thakore, J. H., Mann, J. N, Vlahos, I., et al (2002) Increased visceral fat distribution in drug-naïve and drug-free patients with schizophrenia. International Journal of Obesity and Related Metabolic Disorders, 26, 137-141.
Zhang, Z.-J., Yao, Z.-J., Liu, W., et al (2004) Effects of antipsychotics on fat deposition and changes in leptin and insulin levels. Magnetic resonance imaging study of previously untreated people with schizophrenia. British Journal of Psychiatry, 2004, 184, 58-62.

Zimmerman, U., Kraus, T., Himmerich, H., et al (2003) Epidemiology, implications and mechanisms underlying drug-induced weight gain in psychiatric patients. Journal of Psychiatric Research, 37, 193-220.

\section{MCQs}

1 Core features of the metabolic syndrome include:

a hyperinsulinaemia

b raised blood pressure

c coronary heart disease

d central obesity

e type I diabetes.

2 People with schizophrenia have an increased rate of:

a premature death

b asthma

c heart disease

d smoking

e rheumatoid arthritis.

3 The following statements are true for schizophrenia: a compared with males, females have a higher prevalence of cigarette smoking

b compared with males, females have a higher prevalence of obesity

c people with schizophrenia begin to smoke at a significantly earlier age than the general population

d people with schizophrenia have lower rates of smoking-related illness

e the majority of people with schizophrenia have no interest in altering their smoking behaviour.

4 Risk factors for osteoporosis with schizophrenia include:

a obesity

$\mathrm{b}$ poor nutrition

c smoking

d antipsychotic treatment

e polydipsia.

5 Atypical antipsychotics are associated with:

a increased smoking rates

b increased weight

c increased blood sugar

d decreased extrapyramidal side-effects

e decreased quality of life.

\section{MCQ answers}

\begin{tabular}{|c|c|c|c|c|}
\hline 1 & 2 & 3 & 4 & 5 \\
\hline a $\mathrm{T}$ & a $\mathrm{T}$ & a F & a F & a F \\
\hline $\mathrm{b} T$ & b F & b $\mathrm{T}$ & b $\mathrm{T}$ & b $\mathrm{T}$ \\
\hline c F & c $\mathrm{T}$ & c F & c $\mathrm{T}$ & c $\mathrm{T}$ \\
\hline $\mathrm{d} T$ & $\mathrm{~d} T$ & d F & $\mathrm{d} T$ & $\mathrm{~d} \mathrm{~T}$ \\
\hline $\mathrm{F}$ & e $F$ & e $F$ & e $\mathrm{T}$ & e F \\
\hline
\end{tabular}

